\title{
Efficacy of the larval parasitoid, Bracon hebetor Say. (Hymenoptera: Braconidae) on the greater wax moth larvae, Galleria mellonella (L.) (Lepidoptera: Pyralidae) under laboratory and field conditions
}

\author{
D. Adly ${ }^{1 *}$ id and W. M. Marzouk ${ }^{2}$
}

\begin{abstract}
The greater wax moth, Galleria mellonella Linnaeus (Lepidoptera: Pyralidae), is considered one of the most important pests effecting honeybee industry. The present study was carried out to evaluate the efficacy of the larval parasitoid, Bracon hebetor Say. (Hymenoptera: Braconidae), on G. mellonella in laboratory, honeybee colonies, and stored wax combs. In the laboratory studies, the pre-ovipositoinal, ovipositional, and post-ovipositional periods of the parasitoid were $0.27 \pm 0.45,20.87 \pm 1.5$, and $4.33 \pm 0.48$ days, respectively. The total number of eggs/female of the parasitoid on the 5 th larval instar of $G$. mellonella reached $71.77 \pm 7.84$ eggs. B. hebetor females paralyze their hosts, the percentage of paralyzed 2nd larval instar of G. mellonella was 30\% and parasitoid could not lay eggs on them, while the percentage of paralyzed 5th larval instar was 100\% and parasitoid could lay eggs. In the field studies, the parasitoid, $B$. hebetor was released in honeybee colonies and stored wax combs to evaluate its efficacy. By releasing the parasitoid, the mean numbers of dead larvae of $G$. mellonella in treated honeybee colonies were greater than in the untreated, $(91.8 \pm 5.319$ and $53.3 \pm 24.373)$ larvae/colony, respectively. Also, releasing of $B$. hebetor against $G$. mellonella in stored wax combs reduced the number of survived G. mellonella larvae in treated storage wax combs to $3.2 \pm 2.38$ than in the untreated (using formic acid) $9.3 \pm 5.52$ larvae/store colonies. This is the first work to study efficacy of the parasitoid, B. hebetor on G. mellonella larvae in honeybee colonies and stored wax combs. The results suggested that the parasitoid had the efficacy to be used for controlling G. mellonella in beehives and stored wax comb in Egypt.
\end{abstract}

Keywords: Bracon hebetor, Galleria mellonella, Honeybee colonies, Infested combs, Biological control

\section{Background}

The honeybee (Apis mellifera L.) is the most useful insect to human. Honeybees are attacked by many diseases and pests which cause weakness of colonies and honey production (Shimanuki and Knox 2000). The greater wax moth, Galleria mellonella Linnaeus (Lepidoptera: Pyralidae), is considered one of the most important pests effecting honeybee industry. It is found in apiaries, honeybee colonies, and stores of beekeepers. In Egypt, its

\footnotetext{
* Correspondence: daliaadly27@hotmail.com

'Biological Control Department, Plant Protection Research Institute,

Agriculture Research Center, Giza, Egypt

Full list of author information is available at the end of the article
}

generated economic loss reached up to $40 \%$. Contamination by the pest was found widely distributed in many wax stores during the autumn and winter months (Williams 1997).

New methods for early diagnosis of certain diseases and pests such as hyper-spectral application technology in the honeybee colonies have been reported (Yones et al. 2019). However, biological control integrated with other tactics can play an efficient role in pest management.

These pests are a particular nuisance for frame hive beekeepers who store empty combs and honeycombs outside the hives for use in future beekeeping. The combs, unprotected by bees, are very vulnerable to the wax moths' infestation. Wax moths are attracted to the 
combs that have been used for brood, where their larvae consume the bees' larval skins and pupal cases left in brood comb. Larvae of G. mellonella can damage wooden boxes as the mature larvae spin tough white cocoons, and gouge depressions in woodwork to attach them (Watkins 2005). Wax moths may live inside the hives; however, strong honeybee colonies will not tolerate them. The moths can be a nuisance, if the colony is weak or there are many uncovered combs where the moths can lay eggs causing considerable damages to combs left unattended by bees, honeybee wax combs in storage places and can cause substantial losses to combs, hive material, and bees in beehives all over the world (Ellis et al. 2013).

Temperature at brood nest ranges from 33 to $36^{\circ} \mathrm{C}$ is actively regulated by bees utilizing different methods (Abou-Shaara et al. 2017). This temperature range represents a challenge to the role of biocontrol agents that can be used inside the hives to control Varroa mites, wax moths, or small hive beetles. Therefore, screening the survival ability of the selected biocontrol agents to hive microclimate is a necessary step to determine the appropriate control agents.

Bracon hebetor Say. (Hymenoptera: Braconidae) is a gregarious, ecto-parasitoid that attacks the larval stage of several species of Lepidoptera. B. hebetor is considered one of the best potential biological control agents for stored-product insects in the family Pyralidae (Brower et al. 1996). B. hebetor females paralyze their hosts, which are typically last-stage larvae in a "wandering" phase, by stinging them, injecting paralytic venom, and ovipositing variable numbers of eggs on or near the surface of paralyzed hosts (Alam et al. 2015).

The primary concern for beekeepers is how to adequately store combs without being destroyed or damaged by the larval stages of G. mellonella. Destruction or damage to combs can also occur within weak hives, which have died or have low populations. The presence of an adequate number of adult bees will prevent wax moth damage.

The objective of this study was to evaluate the efficacy of the parasitoid, B. hebetor against the greater wax moth G. mellonella under laboratory conditions, honeybee colonies, and stored wax combs.

\section{Materials and methods Insect culture}

\section{Rearing of Galleria mellonella (L.)}

Larvae of G. mellonella were collected from infested bee hives, reared in jars (2-kg capacity) until emergence of the moths. Their laid eggs were collected. The rearing was carried out in accordance with the methodology adapted by (Dutky et al. 1964), using an artificial diet modified by (Huang et al. 2010) under the laboratory conditions of $28 \pm{ }^{\circ} \mathrm{C}, \quad 60 \% \quad$ R.H., and $16: 8 \mathrm{~L}: \mathrm{D}$ photoperiod.

\section{Rearing of the parasitoid, Bracon hebetor Say.}

A stock culture of $B$. hebetor was obtained from infested flour with the Mediterranean flour moth, Ephestia kuehniella (Sulz.), collected from warehouses of flour mills, Giza Governorate, Egypt. Infested flour was placed in plastic containers, until emergence of parasitoid's adults. Afterwards, newly emerged females and males were isolated in a separated container, supplied with a drop of honey as food source, and the last larval instar of G. mellonella for laying egg to start the parasitoid culture. The containers were kept in an incubator at $28 \pm 1{ }^{\circ} \mathrm{C}, 60 \%$ R.H. (Farag et al. 2015).

\section{Laboratory studies}

Biological studies were carried out under the laboratory conditions of $\left(28 \pm 1{ }^{\circ} \mathrm{C}, 60-70 \%\right.$ R.H. and 16:8 L: D photoperiod).

\section{Durations of immature stages (egg-pupa)}

Newly emerged adults of $B$. hebetor were paired (female and male) provided with 10 larvae of 5th larval instar of $G$. mellonella, for $24 \mathrm{~h}$, in a small plastic container $(10 \mathrm{~cm}$ in height, $7 \mathrm{~cm}$ in diameter), containing droplets of honey, covered with white muslin kept in position by rubber bands. The paralyzed larvae were separated individually in Petri dishes. Only one wasp egg was left on each host. The paralyzed larvae were investigated daily, using a stereomicroscope, to record the durations of immature stages from egg to pupa (30 replicates were used).

\section{Longevity}

Newly emerged parasitoid adults were placed individually in small glass vials and fed on droplets of honey until their death. Longevity of each sex was recorded (30 pairs; males and females).

\section{Fecundity}

Newly emerged females of $B$. hebetor were placed with males for $24 \mathrm{~h}$ in a $500-\mathrm{ml}$ plastic jar for mating. The opportunity for mating was provided as $80 \%$ of virgin $B$. hebetor females mate within the first $15 \mathrm{~min}$ of being in the presence of male (Ode et al. 1995; Dabhi 2011). After $24 \mathrm{~h}, B$. hebetor females were separated from the males and introduced individually in a small plastic jar $(10 \mathrm{~cm}$ in height, $7 \mathrm{~cm}$ in diameter), containing 10 of 2nd and last larval instars of G. mellonella, each age individually, covered with white muslin and kept in position by rubber bands. After $24 \mathrm{~h}$, females were carefully transferred to a new small plastic container containing fresh larvae of a given host age. This procedure was repeated until the death of female parasitoids. Number of 
larvae paralyzed and number of eggs on the paralyzed larvae were counted daily, using a stereomicroscope (30 replicates). Total number of laid eggs, periods of pre-oviposition, oviposition, and post-oviposition were recorded.

\section{Field studies}

The experiments were carried out at the apiary of the Bee Research Department, Plant Protection Research Institute, Agriculture Research Center, Giza, Egypt, under the environmental conditions from September to February the winter season of 2018-2019.

\section{Efficacy of releasing $B$. hebetor against $G$. mellonella in honey bee colonies Honey bee colonies}

Six Carniolan (F1) of A. mellifera carnica honey bee colonies, provided with mated hybrid queens, each at the same age, were prepared in a control experiment at the apiary. Each colony, contained $1.125 \mathrm{~kg}$ of adult bees (about 12,300 bees), 2 of sealed and unsealed brood combs, 2 combs of honey and pollen, and one empty fully drawn comb.

\section{Experimental design}

The colonies were randomly divided into 2 groups 3 honeybee colonies each. The first group (treated group) was provided by 100 of 5th larval instar of G. mellonella and 30 female parasitoids of B. hebetor released weekly, starting from September 2018 to February 2019 for each colony. After that, colonies were closed for $6 \mathrm{~h}$ to avoid way out of the parasitoid attracted by the light. The second group (untreated group) was provided weekly only by 100 of 5 th instar larvae of G. mellonella for each colony and used as a control.

\section{Efficacy of releasing B. hebetor against G. mellonella in stored wax combs}

The experiment was carried out at an apiary store, using 6 langstroth hives, each had 10 wax combs. The hives were randomly divided into 2 groups; the first was provided weekly by 30 females of $B$. hebetor hive, while the second was provided by formic acid $60 \%$ hive and used as control. The experiment started in the 1st week of September 2018 until the end of February 2019.

\section{Assayed parameters}

The following parameters were assayed to evaluate the efficacy of releasing the parasitoid $B$. hebetor in the honeybee colonies and in the stored wax combs experiments:

1. Number of dead and/or survived larvae of G. mellonella/colony.
2. Infested areas of G. mellonella larvae $\left(\right.$ inch $\left.^{2}\right) /$ colony measured by using a frame divided into square inches.

3. Number of infested tunnels/colony.

\section{Statistical analysis}

Data were analyzed in a randomized complete block design (ANOVA) by MSTAT-C version 1.41 (Sendecor and Cochran 1980), and using the graph pad PRISMA version 3.03 for windows, software. All means were compared by Duncan's multiple range test at level 0.05 (Duncan 1955).

\section{Results and discussion Laboratory studies}

The total duration period of the immature stages of $B$. hebetor (egg to pupa) was $11.2 \pm 0.85$ days, with a range of 10-12 days. Longevity and total life cycle of $B$. hebetor females were longer than males. The female longevity and total life cycle attained $25.4 \pm 1.3(23-27)$ days and $36.6 \pm$ 1.5 (34-39) days. For males, they were $15.57 \pm 1.1$ (15-18) days and $26.77 \pm 0.86$ (25-28) days, respectively.

These results disagree with (Masood and Hsin 2006) who reported that the females longevity was shorter than males, $17.42 \pm 1.37$ and $19.4 \pm 1.88$ days, respectively, at $28 \pm 0.5{ }^{\circ} \mathrm{C}$ with a photoperiod of $16: 8$ (L: D) and $65 \pm 5 \%$ R.H. and there was insignificant difference in the total pre-adult duration between $B$. hebetor reared on E. kuehniella and G. mellonella. Farag et al. (2015) results agree with the obtained results of this study, as they stated that the immature stages of the B. hebetor lasted $9.42 \pm 0.2$ days, when reared on G. mellonella and the longevity of female was longer than male, $19.11 \pm 1.8$ and $9.2 \pm 66$ days, respectively, at $28 \pm 0.5^{\circ} \mathrm{C}$ and 16:8 (L:D).

The pre-ovipositoinal, ovipositional, and postovipositional periods of the $B$. hebetor females were 0.27 \pm 0.45 , with a range of (0-1) days, $20.87 \pm 1.5$ (19-23) and $4.33 \pm 0.48(4-5)$ days, respectively. The percentage of paralyzed 2nd larval instar of G. mellonella was 30\% and females failed to lay eggs on them, while the percentage of paralyzed 5th larval instar of G. mellonella was $100 \%$ and females succeeded to lay eggs. The total number of eggs/female on the 5th larval instar of $G$. mellonella reached $71.77 \pm 7.84(60-85)$ eggs. Mean fecundity of B. hebetor attained 78.3 eggs/female on G. mellonella and 66.3 eggs/female on E. kuehniella (Masood and Hsin 2006). But (Farag et al. 2015) reported that the total number of eggs deposited by female of $B$. hebetor reached a maximum of 395.11 eggs on $G$. mellonella comparing with 93.5 and 56 eggs on E. kuehniella and Corcyra cephalonica (Stain.), respectively.

Hagstrum and Smittle (1977) mentioned that B. hebetor females preferred to attack the last larvae at a rate 10-fold more than attacking young larvae. Mohammad 
et al. (2017) reported that the highest frequency of paralysis and percent parasitism were found on C. cephalonica, followed by G. mellonella, E. kuehniella, and Plodiainter punctella, while it was the lowest on Spodoptera litura and S. littoralis.

\section{Field studies}

Efficacy of releasing B. hebetor against G. mellonella in honeybee colonies

a. Number of dead larvae of G. mellonella/colony The mean numbers of dead larvae of G. mellonella in treated honeybee colonies were greater than in the untreated ones. The general means of numbers of dead larvae in treated and untreated honeybee colonies were $91.8 \pm 5.319$ and $53.3 \pm 24.373$ larvae/colony, respectively (Table 1 ). Statistical analysis revealed significant differences between the honeybee colonies that treated with the parasitoid and the untreated ones. The highest number of dead larvae of G. mellonella in the treated honeybee colonies was recorded in February 2019, while the lowest was in November 2018 (97.43 \pm 1.46 and $87.6 \pm 0.88$ larvae/colony, respectively). In the untreated colonies (control), the highest number of dead larvae of G. mellonella was in January 2019, and the lowest number was in September 2018 (84 \pm 6.38 and $30 \pm 14.07$ larvae/colony, respectively) (Table 1). The results showed that the parasitoid $B$. hebetor succeeded to suppress high numbers of the larvae of G. mellonella in honeybee colonies.

b. Infested areas of G. mellonella larvae $\left(\right.$ inch $\left.^{2}\right)$ / colony

The infested areas by silk fiber of G. mellonella larvae $\left(\right.$ inch $\left.^{2}\right)$ in treated wax combs were smaller than in the untreated. The general means of the infested areas were $6.7 \pm 2.03$ and $25.9 \pm 20.16$ inch $^{2} /$ colony, respectively (Table 1 ). The infested areas of G. mellonella larvae declined sharply in the treated wax combs from $32.8 \pm 12.48 \mathrm{inch}^{2} /$ colony in September 2018 to $0.075 \pm 0.144 \mathrm{inch}^{2} /$ colony in January 2019, due to the release of the parasitoid. In untreated wax combs, 2 peaks of the infested areas of G. mellonella were recorded. The first peak ( 65.5 $\pm 40.6 \mathrm{inch}^{2} /$ colony) was in September 2018 and the second one $\left(27.0 \pm 17.5 \mathrm{inch}^{2} /\right.$ colony $)$ was in November 2018 (Table 1). The results showed that the parasitoid $B$. hebetor succeeded to decrease the infested areas of G. mellonella larvae in honey bee colonies. Statistical analysis revealed significant differences between the wax combs treated and untreated (control).

c. Number of infested tunnels/colony
The number of infested tunnels caused by $G$. mellonella larvae in treated wax combs was lower than in the untreated. The general means of the infested tunnels were $0.2 \pm 0.15$ and $22.1 \pm 18.46$ tunnel/colony, respectively (Table 1 ). The number of infested tunnels declined sharply in the treated wax combs from $7.1 \pm 0.72$ tunnel/colony in September 2018 to $0.23 \pm 0.25$ tunnel/colony in February 2019, due to the releases of the parasitoid. In the untreated wax combs, it declined gradually and was high until the end of the experiment (14.75 \pm 9.60 tunnel/colony in February 2019). There are significant differences between the numbers of infested tunnels/colony in treated wax combs and in the untreated (control) ones.

\section{Efficacy of releasing B. hebetor against G. mellonella in stored wax combs}

a. Survival of G. mellonella larvae/stored wax combs (without bees)

The mean number of the survived larvae of $G$. mellonella in treated storage wax combs, using $B$. hebetor, was smaller than in the untreated, using formic acid as a positive control. It was $3.2 \pm 2.38$ and $9.3 \pm 5.52$ larvae/store colonies, respectively (Table 2). The number of survived larvae of $G$. mellonella declined gradually in the treated wax combs from $4.75 \pm 0.3$ larvae/store colony in September 2018 to $0.83 \pm 0.9$ larvae/store colony in February 2019, due to releasing the parasitoid. Within the untreated wax combs, the number of larvae fluctuated and the season ended with a high number of larvae (9.75 \pm 15.5 larvae/store colony) (Table 2). There are significant differences between the numbers of larvae of G. mellonella/store colony in treated wax combs and in the untreated (control) ones.

b. Infested areas of wax combs $\left(\right.$ inch $\left.^{2}\right) /$ stored wax combs (without bees)

The infested areas with the Silk fiber of $G$. mellonella larvae $\left(\right.$ inch $^{2}$ ) in treated stored wax combs were smaller than in the untreated. The general means of the infested areas were $1.14 \pm$ 1.48 and $3.8 \pm 3.27 \mathrm{inch}^{2} /$ storage wax combs, respectively (Table 2). The results showed that no infested areas were found in treated stored wax combs from November 2018 to February 2019 compared with the untreated stored wax combs, as there were infested areas in all months, except December and January (Table 2). Statistical analysis revealed significant differences between the wax combs treated and the untreated (control) ones. 
Table 1 Mean numbers of dead larvae, infested areas, and numbers of infested tunnels/colony caused by the greater wax moth, G. mellonella in treated and untreated honeybee colonies

\begin{tabular}{|c|c|c|c|c|c|c|c|}
\hline \multirow[t]{2}{*}{ Month } & \multirow[t]{2}{*}{ Week } & \multicolumn{2}{|c|}{ No. of dead larvae/wax combs } & \multicolumn{2}{|c|}{ Infested areas of larvae $\left(\right.$ inch $\left.^{2}\right) /$ wax combs } & \multicolumn{2}{|c|}{ No. of infested tunnels/wax combs } \\
\hline & & Treated & Untreated & Treated & Untreated & Treated & Untreated \\
\hline \multirow[t]{4}{*}{ September, 2018} & 1st week & $88.0 \pm 2.0$ & 10 & $17.3 \pm 2.52$ & 60 & $13.7 \pm 1.53$ & 75 \\
\hline & 2nd week & $94.7 \pm 1.53$ & 37 & $55 \pm 13.75$ & 87 & $5.3 \pm 0.58$ & 35 \\
\hline & 3rd week & $93.0 \pm 4.0$ & 42 & $55.7 \pm 35.44$ & 104 & $8.3 \pm 2.89$ & 85 \\
\hline & 4th week & $90.0 \pm 2.0$ & 31 & $3.3 \pm 0.58$ & 11 & $1.0 \pm 1.0$ & 42 \\
\hline Mean/month & & $91.43 \pm 0.95^{\mathrm{B}}$ & $30 \pm 14.07$ & $32.8 \pm 12.48^{\mathrm{A}}$ & $65.5 \pm 40.6$ & $7.1 \pm 0.72^{\mathrm{A}}$ & $59.25 \pm 24.47$ \\
\hline \multirow[t]{4}{*}{ October } & 1st week & $83.0 \pm 3.0$ & 30 & $0.3 \pm 0.58$ & 15 & $2.0 \pm 2.0$ & 11 \\
\hline & 2nd week & $83.0 \pm 3.61$ & 34 & $1.7 \pm 2.89$ & 10 & $1.3 \pm 1.15$ & 18 \\
\hline & 3rd week & $91.3 \pm 2.52$ & 42 & $2.3 \pm 0.58$ & 33 & $2.3 \pm 1.53$ & 35 \\
\hline & 4th week & $95.3 \pm 2.08$ & 31 & $2.7 \pm 4.62$ & 18 & $1.7 \pm 2.08$ & 10 \\
\hline Mean/month & & $88.15 \pm 1.89^{B C}$ & $34.25 \pm 5.44$ & $1.75 \pm 1.15^{\mathrm{BC}}$ & $19.0 \pm 9.9$ & $1.83 \pm 0.3^{8}$ & $18.5 \pm 11.56$ \\
\hline \multirow[t]{4}{*}{ November } & 1st week & $88.7 \pm 1.53$ & 32 & $5 \pm 4.58$ & 16 & $0.0 \pm 0.0$ & 11 \\
\hline & 2nd week & $89.7 \pm 0.58$ & 40 & $14.0 \pm 7.55$ & 53 & $1.7 \pm 2.89$ & 26 \\
\hline & 3rd week & $87.3 \pm 3.06$ & 48 & $0.0 \pm 0.0$ & 21 & $0.3 \pm 0.58$ & 10 \\
\hline & 4th week & $84.7 \pm 3.21$ & 29 & $0.7 \pm 1.15$ & 18 & $0.3 \pm 0.58$ & 12 \\
\hline Mean/month & & $87.6 \pm 0.88^{C}$ & $37.25 \pm 8.54$ & $4.93 \pm 1.01^{B}$ & $27.0 \pm 17.5$ & $0.6 \pm 0.58^{c}$ & $14.75 \pm 7.54$ \\
\hline \multirow[t]{4}{*}{ December } & 1st week & $84.7 \pm 2.08$ & 54 & $0 \pm 0.0$ & 20 & $0.3 \pm 0.58$ & 12 \\
\hline & 2nd week & $85.7 \pm 3.06$ & 32 & $0.7 \pm 1.15$ & 27 & $0.0 \pm 0.0$ & 16 \\
\hline & 3rd week & $95.7 \pm 1.53$ & 54 & $0 \pm 0.0$ & 29 & $0.0 \pm 0.0$ & 20 \\
\hline & 4th week & $99.3 \pm 1.15$ & 82 & $0 \pm 0.0$ & 2 & $0.0 \pm 0.0$ & 18 \\
\hline Mean/month & & $91.35 \pm 0.72^{B}$ & $55.5 \pm 20.49$ & $0.18 \pm 0.29^{C}$ & $19.5 \pm 12.3$ & $0.1 \pm 0.14^{\mathrm{D}}$ & $16.5 \pm 3.42$ \\
\hline \multirow[t]{4}{*}{ January, 2019} & 1st week & $93.3 \pm 4.04$ & 79 & $0 \pm 0.0$ & 22 & $0.0 \pm 0.0$ & 11 \\
\hline & 2nd week & $93.3 \pm 6.11$ & 80 & $0.3 \pm 0.58$ & 13 & $0.0 \pm 0.0$ & 14 \\
\hline & 3rd week & $100.0 \pm 0.00$ & 93 & $0 \pm 0.0$ & 4 & $0.0 \pm 0.0$ & 7 \\
\hline & 4th week & $92.7 \pm 2.52$ & 84 & $0 \pm 0.0$ & 2 & $0.0 \pm 0.0$ & 4 \\
\hline Mean/month & & $94.83 \pm 1.13^{B C}$ & $84 \pm 6.38$ & $0.075 \pm 0.144^{C}$ & $10.3 \pm 9.2$ & $0.0 \pm 0.0^{\mathrm{D}}$ & $9 \pm 4.40$ \\
\hline \multirow[t]{4}{*}{ February } & 1st week & $97.3 \pm 2.52$ & 77 & $0 \pm 0.0$ & 25 & $0.3 \pm 0.58$ & 23 \\
\hline & 2nd week & $98.0 \pm 2.0$ & 67 & $1.3 \pm 1.15$ & 13 & $0.3 \pm 0.58$ & 23 \\
\hline & 3rd week & $94.7 \pm 2.52$ & 81 & $0.3 \pm 0.58$ & 12 & $0.0 \pm 0.0$ & 8 \\
\hline & 4th week & $99.7 \pm 0.58$ & 91 & $0 \pm 0.0$ & 8 & $0.3 \pm 0.58$ & 5 \\
\hline Mean/month & & $97.43 \pm 1.46^{\mathrm{A}}$ & $79 \pm 9.93$ & $0.4 \pm 0.144^{C}$ & $14.5 \pm 7.33$ & $0.23 \pm 0.25^{\mathrm{D}}$ & $14.75 \pm 9.60$ \\
\hline General mean & & $91.8 \pm 3.79^{\mathrm{a}}$ & $53.3 \pm 23.54^{b}$ & $6.7 \pm 2.03^{b}$ & $25.9 \pm 20.16^{\mathrm{a}}$ & $0.2 \pm 0.15^{b}$ & $22.1 \pm 18.46^{\mathrm{a}}$ \\
\hline L.S.D $(0.05)$ & & 3.341 & & 2.43 & & 0.328 & \\
\hline
\end{tabular}

Means in the same row or the same column which have the same letter, are not significantly differed at 0.05 level of probability

\section{c. No. of infested tunnels/stored wax combs (without bees)}

The general mean number of infested tunnels caused by G. mellonella larvae in treated wax combs was lower than in the untreated (positive control using formic acid). It was $1.6 \pm 0.12$ and $5.5 \pm 3.0$ tunnels/colony, respectively (Table 2). The number of infested tunnels declined gradually in the treated wax combs from $3.4 \pm$ 0.8 tunnels/colony in September 2018 to $0.3 \pm 0.3$ tunnels/colony in February 2019, due to releasing the parasitoid. Number of the untreated wax combs declined gradually but the number of infested tunnels increased to $6.0 \pm 11.3$ tunnels/colony at the end of experiment in February 2019 (Table 2). Statistical analysis revealed significant differences between the numbers of infested tunnels/colony in treated wax combs and in the untreated (control) ones.

The present study was carried out (from early September to the end February) during the active season of $G$. 
Table 2 Mean numbers of dead larvae, infested areas, and numbers of infested tunnels/colony caused by the greater wax moth, G. mellonella in treated and untreated stored wax combs

\begin{tabular}{|c|c|c|c|c|c|c|c|}
\hline \multirow[t]{2}{*}{ Month } & \multirow[t]{2}{*}{ Week } & \multicolumn{2}{|c|}{ No. of survival larvae/wax combs } & \multicolumn{2}{|c|}{ Infested areas of larvae $\left(\right.$ inch $\left.^{2}\right) /$ wax combs } & \multicolumn{2}{|c|}{ No. of infested tunnels/wax combs } \\
\hline & & Treated & Untreated (formic acid) & Treated & Untreated (formic acid) & Treated & Untreated (formic acid) \\
\hline \multirow[t]{4}{*}{ September, 2018} & 1st week & $5.3 \pm 2.5$ & 12 & $5.0 \pm 3.6$ & 7 & $2.3 \pm 2.5$ & 5 \\
\hline & 2nd week & $5 \pm 1.0$ & 5 & $4.7 \pm 0.6$ & 3 & $5.3 \pm 0.6$ & 3 \\
\hline & 3rd week & $3.3 \pm 3.5$ & 8 & $1.3 \pm 0.6$ & 4 & $1.0 \pm 1.0$ & 3 \\
\hline & 4th week & $5.3 \pm 4.7$ & 20 & $3.0 \pm 5.2$ & 15 & $5.0 \pm 4.4$ & 20 \\
\hline Mean/month & & $4.75 \pm 0.3^{\mathrm{A}}$ & $11.25 \pm 6.5$ & $3.5 \pm 0.9^{\mathrm{A}}$ & $7.3 \pm 5.4$ & $3.4 \pm 0.8^{\mathrm{A}}$ & $7.8 \pm 8.2$ \\
\hline \multirow[t]{4}{*}{ October } & 1st week & $9.7 \pm 4.5$ & 11 & $5.0 \pm 5.0$ & 12 & $6.0 \pm 5.2$ & 9 \\
\hline & 2nd week & $5.3 \pm 2.5$ & 8 & $0.0 \pm 0.0$ & 3 & $0.7 \pm 0.6$ & 0 \\
\hline & 3rd week & $3.7 \pm 4.7$ & 11 & $4.0 \pm 3.6$ & 7 & $3.0 \pm 3.6$ & 5 \\
\hline & 4th week & $5 \pm 5.0$ & 23 & $1.0 \pm 1.7$ & 10 & $3.0 \pm 3.0$ & 23 \\
\hline Mean/month & & $5.9 \pm 3.1^{\mathrm{A}}$ & $13.25 \pm 6.7$ & $2.5 \pm 2.5^{\mathrm{A}}$ & $8.0 \pm 3.9$ & $3.2 \pm 0.4^{\mathrm{A}}$ & $9.3 \pm 9.9$ \\
\hline \multirow[t]{4}{*}{ November } & 1st week & $0.0 \pm 0.0$ & 10 & $0.0 \pm 0.0$ & 1 & $0.0 \pm 0.0$ & 7 \\
\hline & 2nd week & $3.7 \pm 4.6$ & 13 & $0.0 \pm 0.0$ & 2 & $2.3 \pm 2.1$ & 0 \\
\hline & 3rd week & $12.7 \pm 3.1$ & 21 & $0.0 \pm 0.0$ & 0 & $0.3 \pm 0.6$ & 0 \\
\hline & 4th week & $5 \pm 5.0$ & 20 & $1.0 \pm 1.7$ & 12 & $3.0 \pm 3.0$ & 18 \\
\hline Mean/month & & $5.3 \pm 2.0^{\mathrm{A}}$ & $16 \pm 5.4$ & $0.3 \pm 0.4^{B}$ & $3.8 \pm 5.6$ & $1.4 \pm 0.1^{B}$ & $6.3 \pm 8.5$ \\
\hline \multirow[t]{4}{*}{ December } & 1st week & $0 \pm 0.0$ & 10 & $0.0 \pm 0.0$ & 1 & $0.0 \pm 0.0$ & 7 \\
\hline & 2nd week & $2.3 \pm 1.2$ & 1 & $0.0 \pm 0.0$ & 0 & $2.3 \pm 1.2$ & 1 \\
\hline & 3rd week & $0.3 \pm 0.6$ & 4 & $0.0 \pm 0.0$ & 2 & $0.0 \pm 0.0$ & 4 \\
\hline & 4th week & $1 \pm 1.0$ & 0 & $0.0 \pm 0.0$ & 0 & $0.0 \pm 0.0$ & 0 \\
\hline Mean/month & & $0.9 \pm 0.1^{B}$ & $3.75 \pm 4.5$ & $0.0 \pm 0.0^{B}$ & $0.8 \pm 1.0$ & $0.6 \pm 0.3^{C}$ & $3.0 \pm 3.2$ \\
\hline \multirow[t]{4}{*}{ January, 2019} & 1st week & $1.3 \pm 0.6$ & 2 & $0.3 \pm 0.6$ & 0 & $0.0 \pm 0.0$ & 0 \\
\hline & 2nd week & $0 \pm 0.0$ & 5 & $0.0 \pm 0.0$ & 0 & $0.0 \pm 0.0$ & 4 \\
\hline & 3rd week & $2.7 \pm 4.6$ & 0 & $0.7 \pm 1.2$ & 0 & $2.7 \pm 4.6$ & 0 \\
\hline & 4th week & $1.7 \pm 2.9$ & 0 & $0.0 \pm 0.0$ & 0 & $0.0 \pm 0.0$ & 0 \\
\hline Mean/month & & $1.42 \pm 1.1^{B}$ & $1.75 \pm 2.4$ & $0.3 \pm 0.4^{B}$ & $0.0 \pm 0.0$ & $0.7 \pm 1.2^{c}$ & $1.0 \pm 2.0$ \\
\hline \multirow[t]{4}{*}{ February } & 1st week & $0.3 \pm 0.6$ & 2 & $0.0 \pm 0.0$ & 0 & $0.3 \pm 0.6$ & 0 \\
\hline & 2nd week & $2 \pm 2.0$ & 33 & $1.3 \pm 1.2$ & 13 & $0.3 \pm 0.6$ & 23 \\
\hline & 3rd week & $0.7 \pm 1.2$ & 3 & $0.0 \pm 0.0$ & 0 & $0.0 \pm 0.0$ & 0 \\
\hline & 4th week & $0.3 \pm 0.6$ & 1 & $0.0 \pm 0.0$ & 0 & $0.3 \pm 0.6$ & 1 \\
\hline Mean/month & & $0.83 \pm 0.9^{B}$ & $9.75 \pm 15.5$ & $0.3 \pm 0.3^{B}$ & $3.3 \pm 6.5$ & $0.3 \pm 0.3^{C}$ & $6.0 \pm 11.3$ \\
\hline General mean & & $3.2 \pm 2.38^{b}$ & $9.3 \pm 5.52^{a}$ & $1.1 \pm 1.48^{b}$ & $3.8 \pm 3.27^{\mathrm{a}}$ & $1.6 \pm 0.12^{b}$ & $5.5 \pm 3.0^{\mathrm{a}}$ \\
\hline L.S.D (0.05) & & 0.965 & & 0.61 & & 0.499 & \\
\hline
\end{tabular}

Means in the same row or the same column which have the same letter, are not significantly differed at 0.05 level of probability

mellonella individuals in the apiaries, inner honeybee colonies, and stored wax combs. Obtained results agree with that of Chaudhy (1969) who reported that the greater of wax moth individuals started to appear in pronouncing figures in the apiaries in the spring season and gradually increased in autumn season (September). These numbers were exponentially increased throughout the followed months to reach the highest peak in the winter months.

The results of using the parasitoid $B$. hebetor to control the wax moth in the honeybee colonies and stored colonies helped to reduce the population of larvae, the infested areas of the wax comb, and the number of tunnels made by G. mellonella larvae. This is may be due to the effect of venom of the parasitoid to anesthesia larvae of the wax moth. This corresponds with Ghimire and Phillips (2010) who studied the suitability of 12 potential lepidopteran host species (wax moth one of them) for the development and reproduction of $B$. hebetor and reported that the parasitoid's females can use a wide range of lepidopteran hosts for paralyzing and oviposition. 
However, B. hebetor cannot necessarily develop and reproduce on all host species that paralyze and oviposit on, and optimum reproduction was in the storedproduct pyralid hosts.

Charles et al. (2017) reported that there was no confirmed experiment by using the parasitoids (Apanteles galleriae and B. hebetor (Hymenoptera: Braconidae) of either closed indoor or open field trials against the wax moth. Also, there is a need to establish an ecological interaction between these parasitoids and the wax moth larvae and/or the honeybee colonies, so as to ascertain their usefulness. In addition, there are no confirmed cases of either closed indoor or open field trials of the parasitoid against the wax moth.

Therefore, to the best of our knowledge, this is the first work to study the efficacy of the parasitoid B. hebetor on the G. mellonella larvae in honeybee colonies and stored wax combs.

\section{Conclusion}

The present study showed that the parasitoid B. hebetor had the potential to paralyze and parasitize successfully the 5th larval instar of the greater wax moth G. mellonella in the laboratory, bee hive, and stored wax comb. The results suggested that the parasitoid had the efficacy to be used for controlling G. mellonella in bee hives and stored wax comb in Egypt.

\section{Acknowledgements}

Not applicable

\section{Authors' contributions}

All of the authors of this manuscript contributed equally to the design and/ or execution of the experiments described in the manuscript. Both authors read and approved the final manuscript.

\section{Funding}

This work was not supported by any funding body, but personally financed.

\section{Availability of data and materials}

All data are available in the manuscript.

Ethics approval and consent to participate

Not applicable

\section{Consent for publication}

Not applicable

\section{Competing interests}

The authors declare that they have no competing interests.

\section{Author details}

${ }^{1}$ Biological Control Department, Plant Protection Research Institute, Agriculture Research Center, Giza, Egypt. ${ }^{2}$ Bee Research Department, Plant Protection Research Institute, Agriculture Research Center, Giza, Egypt.

Received: 12 October 2019 Accepted: 26 November 2019 Published online: 16 December 2019

\section{References}

Abou-Shaara HF, Owayss AA, Ibrahim YY, Basuny NK (2017) A review of impacts of temperature and relative humidity on various activities of honey bees. Insect Soc 64:455-446
Alam M, Alam S, Miah M, Mian M, Hossain M (2015) Mass rearing of Bracon hebetor (Hym.: Braconidae) on wax moth, Galleria mellonella (Lep.: Pyralidae) with varying density of parasitoid and the host. J Crop Prot 5:39-48

Brower JH, Smith L, Vail PV, Flinn PW (1996) Biological control, pp. 223-286. In B. Sub ramanyam and D.W. Hagstrum (eds.), integrated management of insects in stored products. Marcel Dekker, New York.

Charles AK, George OO, Paul NN, Suresh KR, Ayuka TF (2017) The biology and control of the greater wax moth, Galleria mellonella. Insects, 17 pp.

Chaudhy SA (1969) Flora of Lyallpur and the adjacent canal -colony districts. West Pakistan Agric - Uni Lyallpur, Pakistan.

Dabhi MR (2011) Comparative biology of Bracon hebetor Say on seven lepidopteran hosts. Karnataka J Agric Sci 24(4):549-550

Duncan DB (1955) Multiple rang and multiple F - test. Biometrics 111:1-24

Dutky SR, Thompson JV, Cantwell GE (1964) A technique for the mass propagation of the DD-136 nematode. J Insect Pathol 6:417-422

Ellis JD, Graham JR, Mortensen A (2013) Standard methods for wax moth research. J. Apic. Res. 52:1-17

Farag NA, Ismail IA, Elbehery HHA, Abdel-Rahman RS, Abdel-Raheem MA (2015) Life table of Bracon hebetor Say. (Hymenoptera: Braconidae) reared on different hosts. Int. J. Chem Tech Res 8(9):123-130

Ghimire MN, Phillips TW (2010) Suitability of different lepidopteran host species for development of Bracon hebetor (Hymenoptera: Braconidae). Environ. Entomol. 39:449-458. https://doi.org/10.1603/EN09213

Hagstrum DW, Smittle BJ (1977) Host finding ability of Bracon hebetor and its influence upon adult parasite survival and fecundity. Environ Entomol 6:437439

Huang CH, Pan XH, Huang DF, Wang BH, Wei GL (2010) Screening of artificial feed formulae for Galleria mellonella L. Gusnhci Agricultural Sciences 41(7): 672-674

Masood AM, Hsin C (2006) Demography of Habrobracon hebetor (Hymenoptera: Braconidae) on Two Pyralid Hosts (Lepidoptera: Pyralidae). Ann Entomol Soc Am 99(1):84-90

Mohammad M, Ansari MS, Hasan F (2017) Paralysis and parasitization potential of Bracon hebetor on various Lepidopteran hosts. Agric Sci Digest 37(4):327328. https://doi.org/10.18805/ag.D-4605

Ode PJ, Antolin MF, Strand MR (1995) Brood mate avoidance in the parasitic wasp Bracon hebetor. Say Anim Behav 49:1239-1248

Sendecor GW, Cochran WG (1980) Statistical methods. The lowa State Univ., press Amer., USA, 7thed

Shimanuki H, Knox DA (2000) Diagnosis of honey bee diseases. U.S. Department of Agriculture, Agriculture Handbook No.AH-690, 61 pp.

Watkins M (2005) Control of wax moth, physical, chemical and biological methods. Amer Bee J 144(3):702

Williams JL (1997) Insects: Lepidoptera (moths) In: Morse R., Flottum K., editors. Honey bee pests, predators, and diseases. Al Root Company; Medina, $\mathrm{OH}$, USA: 121-141 pp.

Yones MS, Ma'moun SAM, Farag MA, Abd El-Raouf MM (2019) Hyper spectral application for early diagnosis of American foulbrood disease in the honeybee (Apis mellifera L.) larvae. The Egyptian Journal of Remote Sensing and Space Science.

\section{Publisher's Note}

Springer Nature remains neutral with regard to jurisdictional claims in published maps and institutional affiliations. 